\title{
Evaluation of left atrial myocardial deformation in patients with acute MR after STEMI using CMR feature tracking
}

Tomas Lapinskas ${ }^{*}$, Laura Urbonaite ${ }^{1}$, Paulius Bucius $^{2}$, Augustinas P Fedaravicius $^{2}$, Agnieta Stabinskaite $^{1}$, Marta Ejsmont ${ }^{2}$, Antanas Jankauskas ${ }^{3}$, Remigijus Zaliunas ${ }^{1}$

From 19th Annual SCMR Scientific Sessions

Los Angeles, CA, USA. 27-30 January 2016

\section{Background}

Left atrium (LA) is an important predictor of cardiovascular morbidity and mortality. Data related to LA functional changes during acute MR after ST-segment elevation myocardial infarction (STEMI) are limited. CMR is rapidly evolving imaging modality and feature tracking becomes very promising technique for assessment of myocardial deformation. The aim of this study was to investigate LA functional changes during acute MR in patients with STEMI using cardiac magnetic resonance $(\mathrm{CMR})$ feature tracking.

\section{Methods}

A total of 30 participants (mean age 59 years; $70 \%$ male) were enrolled into the study and underwent CMR at 1.5 Tesla (Siemens Magnetom Aera). LA volumetric and myocardial deformation parameters were obtained from two- and four-chamber b-SSFP cine images. LA strain (passive strain $\left(\varepsilon_{\mathrm{e}}\right)$, corresponding to atrial conduit phase, active strain $\left(\varepsilon_{\mathrm{a}}\right)$, corresponding to atrial booster pump phase and total strain $\left(\varepsilon_{\mathrm{s}}\right)$, corresponding to atrial reservoir phase) and SR (peak positive strain rate (SRs), corresponding to atrial reservoir phase, peak early negative strain rate (SRe), corresponding to atrial conduit phase and peak late negative strain rate (SRa), corresponding to atrial booster pump phase) were calculated.

\section{Results}

All LA strain parameters were significantly increased in mild MR patients, but not in moderate MR or controls (total strain $\left(\varepsilon_{\mathrm{s}}\right)$ : mild MR $34.1 \% \pm 6.6$, moderate MR $25.7 \% \pm 6.7$, controls $25.0 \% \pm 8.1, \mathrm{p}<0.01$; passive strain $\left(\varepsilon_{\mathrm{e}}\right)$ : mild MR $18.1 \% \pm 3.8$, moderate MR $13.8 \% \pm$ 5.5 , controls $12.6 \% \pm 5.7, \mathrm{p}<0.05$; and active strain $\left(\varepsilon_{\mathrm{a}}\right)$ :

Table 1 Comparison of LA strain ( $\varepsilon$ ) and strain rate (SR) parameters MR population and controls

\begin{tabular}{|c|c|c|c|c|c|}
\hline & & Controls $(n=10)$ & Mild MR $(n=10)$ & Moderate MR $(n=10)$ & $P$ value \\
\hline Left atrial function & Left atrial strain (\%) & & & & \\
\hline Reservoir & $\varepsilon s$ & $25.0(8.1)$ & $34.1(6.6)$ & $25.7(6.7)$ & 0.009 \\
\hline Conduit & $\varepsilon e$ & $12.6(5.7)$ & $18.1(3.8)$ & $13.8(5.5)$ & 0.026 \\
\hline \multirow[t]{2}{*}{ Booster pump } & $\varepsilon a$ & $12.3(3.2)$ & $16.0(4.7)$ & $11.8(3.7)$ & 0.041 \\
\hline & Left atrial strain rate (s-1 & & & & \\
\hline Reservoir & SRs & $1.1(0.2)$ & $1.2(0.1)$ & $1.0(0.2)$ & 0.093 \\
\hline Conduit & SRe & $-0.5(0.2)$ & $-0.7(0.1)$ & $-0.6(0.2)$ & 0.104 \\
\hline Booster pump & SRa & $-1.0(0.2)$ & $-1.1(0.4)$ & $-0.9(0.3)$ & 0.738 \\
\hline
\end{tabular}

$\varepsilon$, strain; $S R$, strain rate; $M R$, mitral regurgitation. Bold $p$ values indicate a significance level $<0.05$. 
mild MR $16.0 \% \pm 4.7$, moderate MR $11.8 \% \pm 3.7$, controls $12.3 \% \pm 3.2, \mathrm{p}<0.05)$. LA strain rate parameters did not reach statistical significance. Intraclass correlation coefficient analysis revealed strong interobserver agreement for all LA strain and strain rate parameters.

\section{Conclusions}

LA longitudinal deformation is enhanced during acute MR in patients with STEMI, but only when MR is mild. CMR feature tracking is highly reproducible, less time consuming and potentially valuable tool for clinical and research applications.

\section{Authors' details}

${ }^{1}$ Department of Cardiology, Lithuanian University of Health Sciences, Kaunas, Lithuania. ${ }^{2}$ Faculty of Medicine, Lithuanian University of Health Sciences, Kaunas, Lithuania. ${ }^{3}$ Department of Radiology, Lithuanian University of Health Sciences, Kaunas, Lithuania.

Published: 27 January 2016

- Convenient online submission

- Thorough peer review

- No space constraints or color figure charges

- Immediate publication on acceptance

- Inclusion in PubMed, CAS, Scopus and Google Scholar

- Research which is freely available for redistribution 